\title{
Estudio de la traducción del chino al español de Sutra del estrado
}

\section{Analysis of the Spanish Version of the Chinese Text of the Platform Sutra}

\section{Xuanzi Cao y Montserrat Franquesa}

\section{Universitat Autònoma de Barcelona}

xuanzi.cao@e-campus.uab.cat / Montserrat.Franquesa@uab.cat

Recibido: febrero-2020. Revisado: abril-2020. Aceptado: mayo-2020.

Resumen: El Sutra del estrado, libro de referencia del budismo con más de mil años de antigüedad y atribuido a Hui Neng (638-713), fue traducido por primera vez al inglés en 1933 por Wong Mou-Lam. El manuscrito más antiguo había sido descubierto en 1900 en Dun Huang por el arqueólogo inglés Auren Stein. La primera versión española a partir de ese original se debe a Laureano Ramírez Bellerín (Hui 2000). Las técnicas utilizadas merecen un análisis riguroso, debido a la falta de términos budistas en español y a la dificultad que supone transcribir del sánscrito. En un aspecto más amplio, el análisis comparativo que presentamos en este artículo no es ajeno al contexto de la globalización, donde el contacto entre culturas y las influencias recíprocas se han convertido en ámbitos de estudio necesarios e indispensables para comprender el mundo en que vivimos.

1. Este artículo se inscribe en el Grup d'Estudi de la Traducció Catalana Contemporània (GETCC) (2017, SGR 1155), reconocido por la Agència de Gestió i Ajuts Universitaris de la Generalitat de Catalunya, y en la «Red de estudios y datos sobre la edición iberoamericana y transnacional (RED-EDIT)» (RED2018-102343-T), financiada por el Ministerio de Economía y Competitividad.

Xuanzi CAO y Montserrat FRANQUESA Estudio de la traducción del chino al español de Sutra del estrado

\section{CLINA}

vol. 6-1, June 2020, 109-125

elSSN: 2444-1961

Ediciones Universidad de Salamanca - CC BY-NC-ND 
Palabras clave: Sutra del estrado; traducción del chino al español; budismo; interculturalidad.

\begin{abstract}
The Altar-Sutra, a reference book in Buddhism attributed to Hui Neng (638713) which is more than a thousand years old, was first translated into English in 1933 by Wong Mou-Lam. The oldest manuscript was discovered in 1900 in Dun Huang by the English archaeologist Auren Stein. The first Spanish version was by Laureano Ramírez Bellerín (Hui 2000). Taking into account the lack of Buddhist terms in Spanish and the difficulties associated to transcribing Sanskrit, the translation techniques adopted and the intervention of the translator deserve a rigorous analysis. In a broader aspect, the comparative analysis presented in this article is not unrelated to the context of globalization. The study of cross-cultural contact and reciprocal influences has become necessary to understand the world in which we live.
\end{abstract}

Keywords: The Altar-Sutra; translation from Chinese to Spanish; Buddhism; crosscultural contact.

\title{
1. INTRODUCCIÓN
}

El budismo, una de las tres principales religiones de la humanidad, tuvo su origen en la India, desde donde se fue difundiendo hacia China. A lo largo del tiempo se fue integrando en la cultura y mentalidad chinas, a partir de la incorporación de un gran número de doctrinas del confucianismo y taoísmo en las escrituras budistas. El paso de los textos originales del sánscrito al chino supuso un reto, los traductores se enfrentaron a grandes problemas y reflexionaron sobre ellos. Uno de los primeros testimonios fue redactado por el monje Zhi Qian entre el año 233 y el 253 d. C. y constata que el monje Wei Zhinan se basó en Confucio y Lao-Tse para establecer los criterios de traducción de las escrituras budistas².

Si nos remontamos a los inicios de la historia de la traducción en el continente asiático, vemos que en China la interpretación de textos budistas se desarrolló a partir de la dinastía Han Oriental (25 d. C. - 220 d. C.) hasta la dinastía Song (960 d. C. - 1279 d. C.).

2. 维祇难曰: 《佛言, 依其义不用饰, 取其法不以严。其传经者, 当令易晓, 勿失厥 义, 是则为善。》座中戌曰: “老氏称: ‘美言不信, 信言不美。’仲尼亦云: ‘书不尽言, 言不尽 意。»Wei Zhinan dijo: «el sentido de las escrituras budistas no necesita adornarse, mientras que su doctrina se debe ajustar para que sea más digerible. Si las traducciones no pierden el significado y son fáciles de entender para la gente, son consideradas las mejores». Lao-Tse expresó, en el mismo sentido, que «las palabras elegantes no son sinceras; las palabras sinceras no son elegantes»; y según el sabio Confucio, «el libro no se puede reflejar completamente a través de la palabra, y la palabra no puede reflejar completamente el significado» (Luo y Chen 2015, 22). [La traducción al español es de los autores de este artículo].

Xuanzi CAO y Montserrat FRANQUESA Estudio de la traducción del chino al español de Sutra del estrado
CLINA

vol. 6-1, June 2020, 109-125

elSSN: 2444-1961

Ediciones Universidad de Salamanca - CC BY-NC-ND 
Durante unos mil doscientos años, innumerables investigadores y monjes, tanto nativos como extranjeros, trasvasaron al chino una cantidad considerable de sutras directamente del sánscrito. Entre ellos, destaca el Sutra del estrado, uno de los libros más famosos e influyentes, y el único redactado por alguien originario de China que fue incluido en el Tripitaka (el compendio de obras canónicas de la tradición budista), por lo que fue designado como sutra (término que se reserva en exclusiva para los discursos de Buda). Desde que se tradujo por primera vez al inglés, en 1933, hasta hoy en día, han aparecido incontables versiones en diferentes lenguas. El original se basa en conceptos procedentes tanto del sánscrito como del chino, por lo que resulta extremadamente difícil de entender para los traductores, que no solo deben poseer una competencia excelente de la lengua original y del sánscrito, sino también profundos conocimientos de la religión budista.

La primera edición en castellano del Sutra del estrado apareció en 1990. Obra de Wang Qianhui, fue publicada en Taiwán (Hui 1990) y hoy en día se encuentra ya descatalogada. Es un volumen bilingüe, con notas al final y también un glosario que recoge los términos búdicos más importantes. Se trata de una traducción hecha en base a criterios literalistas ${ }^{3}$. Para nuestro análisis hemos tomado la versión de Ramírez Bellerín, que parte del manuscrito del siglo IX, el más antiguo. Cabe recordar que los originales del Sutra del estrado son diversos, lo cual siempre supone un problema para cualquier traductor. Las ediciones conservadas son las siguientes:

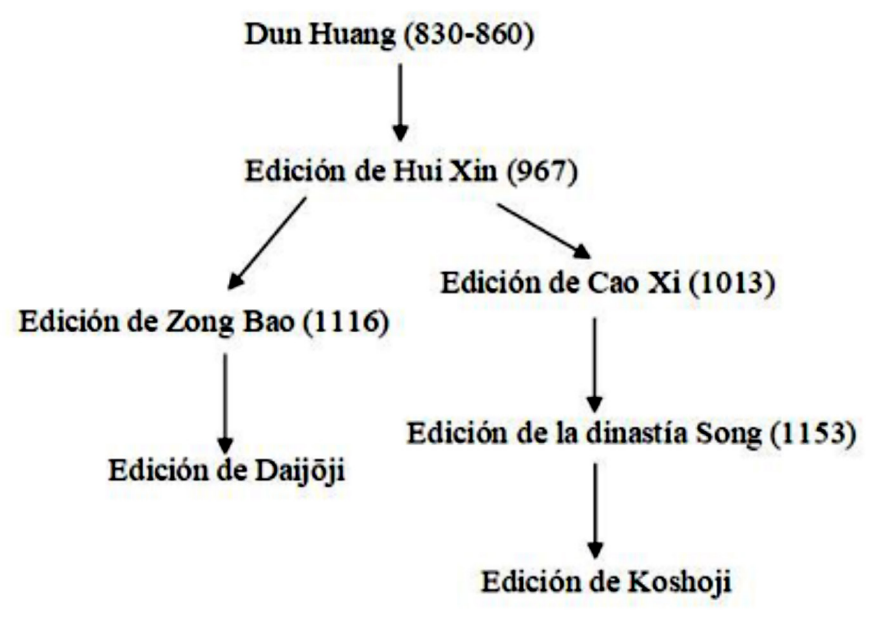

Este artículo tiene por objetivo analizar las técnicas utilizadas por Laureano Ramírez Bellerín en su traducción al castellano. Para situar el alcance de la obra y su contexto,

3. La primera edición es de febrero de 1990, publicada en Taipei. Hemos podido localizar solo un ejemplar en la Biblioteca de Humanidades (Campus de Bellaterra) de la Universidad Autónoma de Barcelona. 
nos remontaremos brevemente al origen del Sutra del estrado y al maestro Hui Neng, cuya obra, compilada por su discípulo, tuvo un papel decisivo para el budismo chan. Nuestro método de análisis va a confrontar fragmentos clave, es decir, pasajes cuyo contenido es fundamental para el budismo, pues incluyen los conceptos en los cuales se basa la ética y las normas de conducta que propugnó Buda. Tratándose de una obra religiosa, que transmite una ideología y cuya lectura puede condicionar creencias o estilos de vida, nos parece de suma importancia estudiar el uso del lenguaje y las opciones del traductor. A través de la comparación entre el original chino y el castellano descubriremos qué criterios le han guiado para asegurar (o no) la exactitud de la versión y conservar (o no) el carácter del original. En un aspecto más amplio, intentaremos descubrir hasta qué punto la traducción ha favorecido, a lo largo de los siglos, el sincretismo religioso característico de Oriente.

\section{EL BUDISMO CHAN}

El budismo llegó a su máximo apogeo en China en la dinastía de Tang (618-907). Fue entonces cuando se dividió en diferentes sectas, entre las cuales una de las más significativas es la denominada chan (禅)4. La creación de esta variante se debe al monje Kāśyapa, uno de los principales discípulos de Buda Gautama. Según la leyenda, en cierta ocasión Buda cogió una flor dorada y la sostuvo en alto. Los discípulos le preguntaron qué significaba, pero él no les explicó nada. Solo uno de ellos, Kāśyapa, consiguió averiguar el significado de ese gesto, hasta entonces misterioso, por lo que Buda le nombró su heredero en la facultad de la enseñanza. Posteriormente, Buda explicó:

Poseo el secreto del ojo verdadero del dharma, de la introspección sutil e inefable en el nirvāna, la que abre la puerta de la visión mística en la forma informe, separada de las palabras y las apariencias, transmitida más allá de las escrituras. Ese secreto debo confiarlo al gran Kāśyapa (Hui 2000, 11).

Por esta razón, Kāśyapa fue reconocido como el primer patriarca hindú. Prajnatara, el vicesimoséptimo, transmitió el dharma a Bodhidarma, quien empezó a introducirlo en China. Bodhidarma se considera el primer patriarca en China y, gracias a él, el chan se transmitió de generación en generación hasta llegar a Hui Neng.

A pesar de que la mayor parte de las escuelas budistas tienen su origen en la India, hay tres que son exclusivamente chinas: la de Tantai, la de Huayan y la de Chan, que han sincretizado el budismo con el pensamiento clásico chino. Puede considerarse

4. Debido a la enorme flexibilidad de los puntos de vista del budismo, se distinguen diferentes sectas, entre las que destacan el budismo theravada, el chan, el tibetano, el de la Tierra Pura y el de nichiren.

Xuanzi CAO y Montserrat FRANQUESA Estudio de la traducción del chino al español de Sutra del estrado
CLINA

vol. 6-1, June 2020, 109-125

elSSN: 2444-1961

Ediciones Universidad de Salamanca - CC BY-NC-ND 
que a partir del segundo patriarca la esencia del budismo incluye el punto de vista de la filosofía del naturalismo, del taoísmo y el estudio moral del confucianismo. Zhang Liwen (1996) sostiene que la sinización del budismo tomó principalmente la filosofía del taoísmo como estructura de base cognitiva. A partir de la dinastía Wei y Jin (220 - 589) empezó a utilizarse el método Ge Yi (traducción sincrética basada en libros clásicos), de manera que el budismo chino integró parte del pensamiento taoísta5

A partir de ahí la escuela Chan se transmitió a Corea y a Japón ${ }^{6}$. En esos países tiene su propio nombre: sôn y zen respectivamente, transliteración del sánscrito dhyāna.

\section{HUI NENG Y EL SUTRA DEL ESTRADO}

El gran maestro y patriarca Hui Neng (638-713) fue uno de los fundadores de la escuela Chan y vivió durante la dinastía Tang, período de pleno apogeo del budismo. Su biografía es una mezcla de mito y realidad. Según consta en el Sutra del estrado, su padre, de apellido Lu, había sido funcionario en Fangyang (en la provincia de Hebei). Posteriormente, su familia se trasladó a Xinzhou, donde nació Hui Neng. La repentina muerte de su padre hizo que tuviera que ocuparse de las tareas domésticas a una edad temprana y que, para mantener a su madre, tuviera incluso que vender leña en el mercado hasta que, un día, un cliente le pidió que le llevara un encargo. Justo cuando se disponía a marchar, se fijó en un hombre que estaba recitando un sutra. En cuanto oyó las palabras de la escritura, su mente se iluminó. Hui Neng preguntó el nombre del sutra y el recitador contestó que se trataba del Sutra del Diamante ${ }^{7}$. En ese momento tomó la determinación de convertirse al budismo. Cuando se enteró de que el patriarca Hong Ren (602-675) estaba predicando ese mismo sutra en el monasterio de Dong Shan, situado en la actual provincia HuBei, fue inmediatamente a encontrarle, tras asegurarse de haber dejado suficiente dinero a su madre para vivir. Una vez allí, no comenzó inmediatamente a estudiar las enseñanzas budistas, sino que durante ocho meses solamente se ocupó de tareas como cortar leña y cocinar hasta el día en el que Hong Ren exigió a los discípulos escribir un gathas con el fin de comprobar si había alguien que se iluminara ${ }^{8}$. Entre todos los discípulos, solo el gathas de Hui Neng

5. Ge Yi se refiere a la utilización de los libros clásicos, tales como Analectas de Confucio, Tao Te Ching o el libro de Zhuangzi, para explicar conceptos del budismo.

6. Fueron ellos quienes llevaron el chan a su país y fue uno de los monjes, Dōshō (629700), quien estableció el budismo japonés zen.

7. Sutra del Diamante, cuyo nombre en sánscrito es Vajracchedikā-prajñāpāramitā-sūtra y en chino 金刚经, Jingang jing, fue traducido por primera vez al chino en el 402 por Kumarajiva (344-413). Es una de las obras más importantes del budismo Mahayana y representa la ideología de la sabiduría, la Prajñā (般若 Banruo), relacionada con la doctrina del Vacío.

8. Gathas (en sánscrito gāthā): cantos o himnos religiosos, generalmente en verso, con o sin rima, pero con repeticiones. Siven para calmar la mente y prepararla para el servicio religioso.

Xuanzi CAO y Montserrat FRANQUESA Estudio de la traducción del chino al español de Sutra del estrado
CLINA

vol. 6-1, June 2020, 109-125

elSSN: 2444-1961

Ediciones Universidad de Salamanca - CC BY-NC-ND 
mostró tener el discernimiento directo de la naturaleza esencial, de manera que Hong Ren le nombró sucesor. Sin embargo, al poco tiempo tuvo que huir al sur de China, perseguido por las envidias que generaba. Permaneció en la clandestinidad durante dieciséis años, hasta el 676, cuando reapareció en el monasterio de Bao Lin (provincia de Cantón). Allí estableció una escuela budista y empezó a enseñar su doctrina.

Gracias a él se popularizó el budismo chan. Su obra capital, el Sutra del estrado, fue compilada por su discípulo, Fahai, de acuerdo con lo que había predicado.

\section{ANÁLISIS COMPARATIVO ENTRE EL ORIGINAL CHINO Y LA TRADUCCIÓN AL ESPAÑOL DE SUTRA DEL ESTRADO}

Antes de pasar al análisis comparativo, debemos justificar la elección de las muestras, el corpus de trabajo. La principal dificultad se encuentra en que existe más de un original, como apuntábamos anteriormente. Según Yang Zengwen (1988, 104), el Sutra del estrado ha tenido en total unas treinta ediciones a lo largo de la historia y las más valiosas son la de Dun Huang, la de Hui Xin, la de Cao Xi y la de Zong Bao. Esta última, basada en varias ediciones que circulaban en ese momento y publicada durante la dinastía Ming (1368-1644), se consideró la más fiable hasta finales del siglo XIX. En la actualidad la edición más reconocida y popular es la descubierta por el arqueólogo inglés Auren Stein en Dun Huang. Cotejada con las demás, resulta ser la más antigua. Por lo tanto, será el material de nuestro análisis, a pesar de que Philip Yampolsky $(2012,89)$ supone que no sería la más antigua, por las numerosas repeticiones, omisiones y equivocaciones que contiene (Chang y Zhao 2018).

Para nuestro análisis hemos elegido la versión española del que fue durante años profesor de chino en la Facultad de Traducción e Interpretación de la Universidad Autónoma de Barcelona y Premio Nacional de Traducción en 1992, Laureano Ramírez Bellerín. Publicada en el año 2000 (Barcelona: Kairós), con introducción y notas del mismo traductor, incluye el texto original de la edición de Dun Huang al final. Los términos o expresiones seleccionados son los fundamentales, los que contienen los conceptos clave del budismo. A partir de ellos realizaremos el análisis comparativo con el español de aspectos léxicos, estilísticos y culturales. Se trata de localizar exactamente cómo se transmite una determinada manera de pensar. Por ello, centramos nuestro interés en una muestra representativa que contiene un elevado peso conceptual.

TO: 惠能大师于大梵寺讲堂中, 升高座, 说摩诃般若波罗蜜 法，授无相戒。(p. 127)
TM: El gran maestro Hui Neng subió al alto estrado del predicatorio del monasterio del Gran Brahmā para predicar la Doctrina del Mahāprajñāpāramitā y los preceptos de la inmaterialidad. (p. 45)

Ejemplo 1

Xuanzi CAO y Montserrat FRANQUESA Estudio de la traducción del chino al español de Sutra del estrado 
Desde el principio de la obra encontramos que el traductor ha utilizado diferentes técnicas con respecto a los nombres propios y en este breve fragmento podemos observar tres opciones diferentes. En primer lugar, Da Fan Si (大梵寺) se traduce como «el monasterio del Gran Brahmā». Se basa en el original, transcrito del sánscrito Brahmā. Además, en una nota, el traductor apunta la fonética china. Por lo tanto, observamos que Ramírez Bellerín mantiene la literalidad a la vez que asegura la exactitud de la traducción. Podría haber utilizado el Pin Yin, como la versión inglesa de Yampolsky $(2012,125)$, que lo traduce por Ta - Fan, pero no lo hace. Se trata, como es evidente, de dos opciones antagónicas.

En segundo lugar, observemos el término Mo He Ban Ruo Bo Luo Mi (摩词般若 波罗蜜) cuyo original en sánscrito es Mahāprajñāpāramitā. Con el objetivo de reproducirlo en su totalidad, el traductor optó por el préstamo original, insertando una nota al pie. La versión china cita la transcripción de Xuan Zang utilizando la transliteración del sánscrito. Aquí el traductor recurrió directamente a la fuente para evitar confusión.

En tercer lugar, encontramos Wu Xiang Jie (无相戒), «los preceptos de la inmaterialidad». Si analizamos este término, descubrimos que se refiere al pensamiento esencial del budismo, es decir, remite al método de la iluminación, el cual resulta enormemente difícil de explicar. En este caso, la técnica de la paráfrasis consigue transmitir el significado del original.

TO: 汝等门人终日供养, 只求福 田, 不求出离生死苦海。 (p. 129)
TM: Vosotros, mis discípulos, andáis ocupados todo el día en hacer ofrendas, buscando tan sólo el campo de la bienaventuranza y no el escape del océano del padecimiento de la muerte y la vida. (p. 48)

Ejemplo 2

Fu Tian (福田), según la creencia budista, es el término que corresponde al hecho de realizar ofrendas a los monjes y buenas acciones a los demás; así aumenta la propia virtud, de manera que obtendremos la bendición divina y podremos conseguir lo que queramos. Esto se corresponde, en la cultura occidental, con la metáfora de sembrar buenas semillas en primavera, regándolas cuidadosamente, para poder cosechar sus frutos al llegar al otoño, o «el que siembra, recogerá», según el refranero español. En el original sánscrito se trata de la expresión Punya-Ksetra, que concentra muchos sentidos. En este caso, con el fin de expresar claramente su significado y ajustarlo al original, se tradujo del chino por "el campo de la bienaventuranza", recurriendo de alguna manera a la terminología bíblica de «bienaventuranza», del Nuevo Testamento (Mt 5, 3-12). Por lo tanto, podemos considerar que el traductor utiliza la técnica de la adaptación.

TO: 善知识, 静听。惠能慈父, 本贯范阳，左降迁流岭南，作 新州百姓 (p. 127)
TM: Escuchad en calma, amigos en la ley. Mi buen padre, natural de Fangyang (p. 46)

\section{Ejemplo 3}

Xuanzi CAO y Montserrat FRANQUESA Estudio de la traducción del chino al español de Sutra del estrado 
Shan Zhi Shi (善知识), que corresponde al sánscrito Kalyāna-mittatā, significa al pie de la letra «amistad espiritual», el concepto con el que Hui Neng expresa el respeto por sus discípulos. Se trata de una fórmula de cortesía que demuestra la modestia de quien habla, en este caso el sexto patriarca. El traductor lo traslada como «amigos en la Ley», utilizando la técnica del equivalente contextual. La palabra «amigos» define una relación de igualdad entre el maestro y sus discípulos, mientras que "en la ley» se refiere al contexto de las comunidades budistas, regidas por unas normas determinadas.

Hui Neng Ci Fu (惠能慈父) se traduce al español de una manera más simple, pues «mi buen padre» en el original es el «buen padre de Hui Neng». En chino queda expresado de una manera más respetuosa en cuanto se alude al maestro por su nombre propio en lugar de hablar en primera persona. Sin embargo, para facilitar la lectura, el traductor la utiliza. Queda claro que entre el chino y el español existen diferencias culturales y convenciones que afectan al aspecto estilístico.

TO: 善知识，定慧犹如何等? (p. 134)
TM: Amigos en la ley, ¿a qué se parecen la contemplación interna y la sabiduría?

(p. 59)

\section{Ejemplo 4}

Analicemos el vocablo Ding Hui (定慧), pues en realidad abarca dos conceptos: el primero, Ding, se refiere al hecho de poseer un espíritu limpio, libre de cosas mundanas y de las interferencias de preocupaciones banales; el segundo, Hui, remite a la sabiduría, a la capacidad de distinguir entre lo bueno y lo malo. El traductor utilizó la técnica de la descripción, por una parte, y el equivalente acuñado, por otra. En español no existe un término similar a Ding. Una traducción literal podría ser "calma», pero el significado queda incompleto.

TO: 若如是, 此法同无情, 却是 障道因缘。道须流通。 (p. 135)

TO: 开口说是人非, 与道相背, 看心看净，却是障道因缘。 (p. 137)
TM: Si fuera así, esta doctrina sería inhumana y obstruiría las causas de la Verdad. La verdad ha de ser fluida. (p. 58)

TM: Criticando a los demás infringen la verdad. Obstinarse en el corazón y la pureza es causa de obstrucción de la Verdad. (p. 63)

\section{Ejemplo 5}

El primer término subrayado en la versión española, Dao (道), la Verdad, se puede encontrar en las ideologías o éticas tradicionales tales como el taoísmo y el confucianismo, no solo en el budismo. Su explicación tiene matices y en el pensamiento clásico chino se entiende, en general, como el «camino», la «vía» o la «doctrina» y, en cierta 
medida, también es así para el budismo. Dado que en el español no existe ningún término o pensamiento tradicional similar y con el fin de evitar confusiones, el traductor adopta la técnica del equivalente acuñado traduciendo Dao por «verdad».

El segundo que hemos señalado, "causa», Yin Yuan (因缘), proviene del sánscrito Nidana, propuesto por Buda Gautama. En chino hace referencia a una concatenación de causa y efecto: todos los eventos son el resultado de lo hecho anteriormente. En Occidente, la causa es algo diferente, un concepto desarrollado por Aristóteles (Física II, 194b). La causa es el principio del cambio y se considera la matriz del movimiento hacia una consecuencia. Por ello, y, con el fin de adaptarlo a la cultura española, se tradujo también por «causa» usando la técnica del equivalente acuñado.

TO: 此法门中何名坐禅?

(p. 138)
TM: ¿A qué llamamos, según esta doctrina, instalarse en la meditación? (p. 63)

TO: 何名为禅定? (p. 138)

TM: ¿A qué llamamos abstracción en la meditación? (p. 64)

En la enseñanza del budismo chan hay dos términos muy importantes que convergen en la palabra traducida por «meditación»: Zuo Chan (坐禅) y Chan Ding (禅 定). Zuo Chan en una traducción literal significa «sentarse y meditar», acción también expresada con el término Zazen ${ }^{9}$. No obstante, según Ramírez Bellerín, en español es preferible traducir Zen por «aposentarse» o «instalarse» y no literalmente por «sentarse». El traductor lo traduce por «instalarse en la meditación», adaptándose así lo más posible a la lengua de llegada. Podría considerarse como una ampliación.

Chan Ding está basado en dos términos sánscritos: Chan corresponde al sánscrito Dhyāna de acuerdo con la traducción fonética, y es un tipo de meditación, un concepto clave del budismo y del hinduismo. Ding, en sánscrito samādhi, es el estado de la conciencia durante la meditación o contemplación. La versión española, mediante la técnica de traducción del equivalente acuñado, se transcribió por «abstracción en la meditación», es decir, explicitando la práctica budista, para resultar más comprensible.

TO: 一时逐惠能口道，令善知识 见自三身佛。

(p. 138)

TM: Si queréis contemplar, amigos en la ley, el triple cuerpo de Buda que anida en vosotros mismos. (p. 65)

9. Zanzen consta de dos palabras: Zan que significa «sentarse» y Zen, «meditación». El término zen es la versión japonesa de chan transliterado y un concepto ampliamente extendido en la actualidad.

Xuanzi CAO y Montserrat FRANQUESA Estudio de la traducción del chino al español de Sutra del estrado 
TO: 于自色身的依清净法身佛 于自色身的依千百亿化身佛 于自色身的依当来圆满报身佛 (p. 138)
TM: Refugiado y alojado en mi propio cuerpo físico, contemplo la limpieza y pureza del cuerpo espiritual de Buda.

Refugiado y alojado en mi propio cuerpo físico, contemplo las miríadas de manifestaciones del cuerpo revelado de Buda.

Refugiado y alojado en mi propio cuerpo físico, contemplo la plenitud futura del cuerpo beatífico de Buda. (p. 65)

Ejemplo 7

En primer lugar, analicemos el término San Shen Fo (三身佛). La transcripción del sánscrito es Trikāya, y se refiere a los tres cuerpos de Buda o los tres cuerpos de la iluminación, que son los tres aspectos de la realidad del universo: Dharmakaya (法 身, Fa shen), Sambhogakaya (化身, Hua shen) y Nirmanakaya (报身, Bao shen). Se traduce por el «triple cuerpo de Buda», con una traducción literal a partir de la versión china. Sin embargo, si nos detenemos a examinar las traducciones de cada aspecto de Buda, el primero de los San Shen Fo, Dharmakaya, en el Sutra del estrado se tradujo al chino por Fa Shen Fo (法身佛), cuyo significado aproximado podría ser «el cuerpo de la Verdad y la Ley correspondiendo con el vacío y con la mente en su pureza búdica». Por lo tanto, en la versión española, se adopta la técnica del equivalente contextual, traduciendo Fa Shen Fo por «el cuerpo espiritual de Buda». Sambhogakaya pasa al chino como Hua Shen Fo (化身佛), cuyo significado es «la luz que despliega el mundo». En la versión española también se utiliza la técnica del equivalente contextual transcribiéndolo como «el cuerpo revelado de Buda».

En último lugar, observemos el término Nirmanakaya, cuya versión china es Bao Shen Fo (报身佛). Se refiere a un ser, manifestación de Buda, que se materializa para poder transmitir sus enseñanzas a todo el mundo, razón por la cual se traduce por "cuerpo beatífico de Buda», aplicando un equivalente contextual.

Respecto al término Gui Yi (仮依), que en español leemos como «refugiado y alojado", el significado chino proviene de un término en pali, uno de los idiomas índicos, que transcrito sería saranam gacchami. El concepto en español queda traducido a partir de la raíz de «refugio», posiblemente a causa de la influencia inglesa de refuge, que se explica a partir de la idea de que, cuando la gente sufre mucho, se puede refugiar en el budismo. En realidad, el traductor se basa en la versión original mejorándola, pues «refugiado y alojado» completa y ajusta el significado en chino.

TO: 将大智慧到彼岸，打破五阴 TM: Emplead su gran sabiduría para llegar a la otra orilla (al 烦恼尘劳。

(p. 144) nirvana) y para derrotar las aflicciones y las pasiones de los Cinco Componentes de los seres inteligentes. (p. 76)

Ejemplo 8

Xuanzi CAO y Montserrat FRANQUESA

Estudio de la traducción del chino al español de Sutra del estrado
CLINA

vol. 6-1, June 2020, 109-125

elSSN: 2444-1961

Ediciones Universidad de Salamanca - CC BY-NC-ND 
En la traducción de Wu Yin (五阴) se ha utilizado la técnica del equivalente contextual, pues las cinco Skaandhas del sánscrito son las cinco acumulaciones o sustancias que componen un ser inteligente, en referencia al ser humano; es decir, la forma o materia, el cuerpo físico con los cinco órganos de los sentidos, que son los «Cinco Componentes de los seres inteligentes». Por otro lado, si atendemos a la versión china, se habla de los cinco Yin. El concepto Yin para el taoísmo es la oscuridad, la tierra o lo malo, entre otros aspectos. En este caso el traductor no recurrió a la versión china. Para evitar confusiones primó el concepto sánscrito.

TO: 愿自三宝慈悲證明。 (p. 142)
TM: Ojalá que nuestros Tres Tesoros interiores lo testifiquen con piedad.

(p. 72)

Ejemplo 9

El término San Bao corresponde en sánscrito a Triratna y significa «Tres Joyas o Tesoros", los tres valores esenciales del budismo: Buda (Fo, 佛), el Dharma (Fa, 法) y la Sangha (Seng, 僧). La versión española recurre a la técnica de la traducción literal, trasladando directamente el concepto chino, «Tres Tesoros».

TO: 今故远来礼拜和尚, 不求馀 TM: Vengo expresamente para rendiros culto, y no busco

物, 唯求作佛法。(p.128) cosa alguna, tan sólo ser instruido en la Ley de Buda. (p.47)

Ejemplo 10

Fo Fa (佛法), en sánscrito Dharma, significa la doctrina y el dogma representados por la verdad de Buda. Aquí se ha optado por la técnica de la traducción literal del chino, «la Ley de Buda». Según explica Shariputra, uno de los diez principales discípulos de Śākyamuni Buda, en realidad el Dharma tiene gran cantidad de sentidos y se utiliza en contextos diferentes. En este caso, para evitar la confusión de su significado, el traductor opta por la literalidad.

TO: 惠能闻说，宿业有缘，便即 TM: Al oírlo me sentí predestinado por el Karma de mis 辞亲。

(p. 128) existencias anteriores y, despidiéndome de mi madre. ( $p$. 47)

TO: 自是我宿业障重, 不合得 法。(p. 130)

TM: Porque existe alguna obstrucción importante en mis existencias pasadas que me impide acceder a la doctrina. (p. 50)

Ejemplo 11

Xuanzi CAO y Montserrat FRANQUESA Estudio de la traducción del chino al español de Sutra del estrado 
Con la comparación de estos dos pasajes vemos que la traducción del término Su Ye (宿业) se ha resuelto de dos maneras diferentes al español: en el primer caso se mantiene «Karma», en la lengua original, el sánscrito, mediante la técnica del préstamo. En el segundo recuadro la mención al Karma se omite y leemos directamente «obstrucción importante», en chino zhang zhong, (障重). Vemos que existen diferencias entre el texto chino y el español, y en comparación, este último tiende a un estilo más austero y con una mayor economía del lenguaje para evitar las repeticiones. El chino no solo tiene inclinación por los adornos, sino que insiste en mencionar repetidamente objetos y frases ya citados anteriormente, para evitar la indefinición.

$\mathrm{TO}$ : 若争先后, 即是迷人 (p. 134)

$\mathrm{TO}$ : 迷人着法相

(p. 135)

TO: 迷人不悟, 便执成颠倒 (p. 135)
TM: Pues las discusiones en torno a la procedencia de una $u$ otra confunden a las gentes. (p. 57)

TM: Las gentes confundidas se aferran a la apariencia del dharma. (p. 58)

TM: Las gentes desorientadas que intentan cosechar méritos con este proceder no logran la iluminación.

(p. 59)

Ejemplo 12

Mi Ren (迷人) aparece en español de tres maneras diferentes: "confunden a las gentes», «las gentes confundidas» y «las gentes desorientadas». Se trata del mismo término en chino, idioma que repite extraordinariamente. El español, en cambio, expresa de distintas maneras un mismo concepto. Las repeticiones en chino ayudan a la comprensión, como apuntábamos antes, mientras que en español la hacen más difícil.

TO: 付汝衣法, 禀为六代。 (p. 129)
TM: Le pasaré mis hábitos y mi doctrina y lo nombraré Sexto Patriarca. (p. 63)

Ejemplo 13

El término de Yi Fa (衣法) hace referencia a la kāsāya, la ropa de los monjes y a la vez también al dharma (Fo Fa, 佛法), la doctrina o ley natural. Aquí se ha traducido por «hábito» y «doctrina», pues, por una parte, indica el hábito del monje budista (que consta de tres prendas básicas: el antravāsas o «hábito interno»; el uttarāsanga o «hábito externo» y el samghātī o «hábito comunitario») y, por otra, la doctrina. El dharma tiene diversos significados, como hemos citado anteriormente. Se ha optado por la técnica del equivalente contextual, tanto en «hábito» como en «doctrina». 
TO: 《直心是道场, 直心是净 土》莫心行謟曲，口但说直， 口说一行三昧, 一行直心，非 佛教弟子。(p. 135)
TM: «La intuición es el lugar donde Buda alcanzó la iluminación, la intuición es la Tierra Pura» No mencionéis sólo de palabra la intuición, mientras vuestro corazón se pierde en lisonjas y divagaciones, pues aquél que únicamente habla de la unión de la meditación con su objeto sin practicar la intuición no es discípulo. (p. 58)

Ejemplo 14

Zhi Xin (直心) aparece traducido cuatro veces de la misma manera. En chino significa que no debemos hablar mal de los demás, ni halagarlos, sino que debemos tratar con corazón sincero a todas las personas. Literalmente, Zhi Xin es «pensamiento directo». Se utiliza la técnica del equivalente acuñado, pues el vocablo «intuición» es un concepto conocido en la lengua de llegada, de manera que resulta más fácil de entender.

$\mathrm{TO}$ :此是最上乘法, 为大智上根 TM: Es una doctrina suprema, y está concebida para 人说, 少根之人, 若闻法, 心 gentes dotadas de gran conocimiento intuitivo, pues las 不生信。(p. 142) gentes de escasa intuición no la creerán al oírla. (p. 72)

Ejemplo 15

Gen (根), cuyo significado general es «raíz», «base» u «origen» aplicado a los sentidos, se refiere también a un «poder» o «facultad». En el budismo Gen Zhi (根智) corresponde a la sabiduría fundamental, es decir, el conocimiento de los principios básicos o intuitivos. En este caso, el traductor, de acuerdo con la técnica del equivalente acuñado, traduce por «intuición». En el ejemplo anterior se sirvió del mismo vocablo y de la misma técnica para explicar el concepto Zhi Xin (直心), en ambos casos para facilitar la comprensión en la lengua de llegada.

\begin{tabular}{l|l}
$\begin{array}{l}\text { TO: } \\
\text { 身是菩提树, }\end{array}$ & TM: \\
心如明镜台。 & El cuerpo es el Árbol del Bodhi, \\
时时勤拂拭, & el corazón, como el pedestal de un espejo brillante. \\
莫使有尘埃。(p. 130) & No dejes de bruñirlo con afán en todo momento, \\
pO: & para que no tenga polvo. (p. 50) \\
\hline TO: & TM: \\
菩提本无树, & El bodhi no es un árbol, \\
明镜亦无台。 & el espejo brillante no tiene pedestal. \\
佛性常清静, & Si la naturaleza búdica es por siempre pura y limpia, \\
何处有尘埃? (p. 132) & ¿dónde está el polvo? (p. 53) \\
\hline TO: & TM: \\
心是菩提树, & El corazón es el Árbol del Bodhi, \\
身为明镜台。 & el cuerpo como el pedestal de un espejo brillante. \\
明镜本清净, & Si el espejo brillante permanece puro y limpio, \\
何处染尘埃? (p.132) & ¿dónde está el polvo que lo empañe? (p. 53) \\
\hline
\end{tabular}

Ejemplo 16

Xuanzi CAO y Montserrat FRANQUESA

Estudio de la traducción del chino al español de Sutra del estrado 
Estos tres Gatha son los más conocidos de la historia del budismo en China. El primer recuadro corresponde a un texto de Shen Xiu (606-706), uno de los más famosos discípulos del Quinto Patriarca, mientras que los otros dos fueron escritos por Hui Neng. Su fraseología es parecida a la poesía, con una estructura pareada, mientras que su contenido expone las esencias divinas de la verdad, el buen pensamiento y el espíritu de la rectitud que demuestran que una persona ha logrado la iluminación.

La versión española, en comparación con los tres Gathas originales, tiende a la traducción literal y pierde el particular ritmo poético. En realidad, resulta imposible transmitir completamente forma y significado, debido a la peculiaridad del texto, que acumula tanta sabiduría antigua. Ya en la dinastía Jin del Este (317 d. C. - 420 d. C.), el monje Shi Daoan (312-385) expuso, en el prefacio del Sutra de Mahaprajapati-paramita (摩诃般若波罗蜜经抄序), unas reflexiones conocidas como «Tres casos no fáciles»

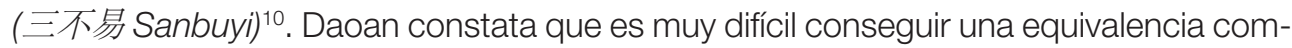
pleta entre la lengua de origen, el sánscrito, y la de llegada, el chino.

Debemos destacar la traducción del término Pu Ti Shu (菩提树), una especie del género Ficus, árbol endémico del Nepal, India y sudoeste de China, bajo el cual, según cuenta la leyenda, Siddhartha Gautama se sentó a meditar y alcanzó el nirvana y la iluminación. Este árbol endémico no se conoce en Occidente ni existe una especie similar para explicarlo. En este caso, el traductor utiliza la técnica del préstamo: «Árbol del Bodhi». Sobre esta cuestión, el monje Zhi Qian ya comentó que «el nombre de los objetos distintos en los diferentes países no será fácil de transmitir» "11 (Chen 2000, 6).

10. 1. 然般若经，三达之心，覆面所演，圣必因时，时俗有易，而删雅古，以适今时，一 不易也。Debido a que los sutras Prajñāpāramitā fueron escritos de acuerdo con las costumbres y el pensamiento de aquella época, para adaptarlos a las costumbres de hoy, el traductor tiene que saber cómo usar la lengua contemporánea para sustituir la antigua, cultivando el hábito de la lectura moderna para poder traducir un texto budista. 2. 愚智天隔, 圣人匡阶, 乃欲以千岁 之上微言, 传使合百王之下末俗。二不易也。La inteligencia del sabio y la del vulgo se encuentran tan distantes como el cielo y el abismo. Los textos sagrados concentran toda la sabiduría de Buda, con el fin de transmitir los significados de los sabios al pueblo vulgar, sin cultura. Sin duda se trata de una tarea muy difícil. 3. 阿难出经, 去佛未远, 尊大迦叶, 令五百六通, 迭察 迭书, 今离干年, 而以近意量截, 彼阿罗汉乃兢敚若此, 此生死人而平平若此, 岂将不知法 者勇乎，斯三不易也。Poco después de que Buda partiera hacia Sukhāvatī, Ānanda anotó sus palabras; Mahākāśyapa mandó a unos quinientos grandes monjes que cotejaran con mucho rigor los textos budistas apuntados por Ānanda. Pero, hasta hoy en día, han pasado mil años, y es imposible que el traductor actual posea la misma actitud arhat (阿罗汉) para traducir las escrituras budistas, pues tiene que sobreentender los sutras de Buda y transcender o interpretar las limitaciones de la instrucción misma. Esto no es fácil (Chen 2000, 10). [La traducción al español es de los autores del artículo].

11. La traducción de la cita es de los autores del artículo (名物不实, 传实不易).

Xuanzi CAO y Montserrat FRANQUESA Estudio de la traducción del chino al español de Sutra del estrado
CLINA

vol. 6-1, June 2020, 109-125

elSSN: 2444-1961

Ediciones Universidad de Salamanca - CC BY-NC-ND 
TO:

众生无边誓愿度。

烦恼无边堑愿度。

法门无边誓愿学。

无上佛道誓愿成。

(p. 140)
TM:

Juro y prometo salvar a los infinitos seres vivientes;

Juro y prometo atajar las infinitas aflicciones;

Juro y prometo estudiar las infinitas puertas de la iluminación Juro y prometo seguir el supremo camino de Buda.

(p. 65)

Ejemplo 17

Respecto al aspecto formal de los gathas, la versión española no logra mantener completamente el ritmo del original, aunque intente buscar una métrica con un efecto similar. Se opta por la repetición al principio de cada verso de la expresión «Juro y prometo» (Shi Yuan, 誓愿), para conseguir un efecto rítmico.

Por otro lado, los dos verbos («jurar»y «prometer») aparecen conjugados en primera persona, mientras que en el texto original no está presente el sujeto. Recordemos que son gathas del maestro Hui Neng y, como hemos explicado en el ejemplo 3, no se utiliza la primera persona por cuestión de respeto y estilo.

Dignos de análisis son los términos subrayados en español Zhong Sheng (众生), Fa Men (法门), y Fo Dao (佛道). El primero, Zhong Sheng, remite al sánscrito Sattava a través de la técnica del equivalente acuñado. Sattava posee ocho significados y, con el fin de evitar confusión, se usó directamente el vocablo chino en la versión española, literalmente «seres vivientes». Sobre Fa Men, cuya traducción española es «las puertas de la iluminación», la técnica adoptada es la traducción literal y el equivalente acuñado. El original Fa Men, «las puertas del dharma», se sustituye por «iluminación» en su versión española. Finalmente, en el caso de Fo Dao se aplica también la traducción literal, «el camino de Buda», que resulta fácil de entender.

\section{CONCLUSIONES}

A partir de diecisiete ejemplos hemos confrontado fragmentos bilingües chino-español que nos han permitido una aproximación a las diferentes técnicas utilizadas por Ramírez Bellerín en la traducción del Sutra del estrado. De nuestro análisis resulta que la mayoría de los términos no tienen palabras equivalentes en la lengua de llegada, por lo que el traductor se ha visto obligado a remitirse al sánscrito original, a traducir literalmente del chino o a agregar exégesis tanto en notas al pie como en el cuerpo del texto para llegar a completar el sentido, modificando inevitablemente el lenguaje original y creando algunas palabras nuevas. Las técnicas más utilizadas son el equivalente acuñado, la traducción literal y el préstamo, una prueba, a nuestro entender, de que el budismo en España, al menos al final de la década de los noventa, todavía se encuentra en una primera etapa de desarrollo y no cuenta con suficiente vocabulario. La falta de léxico ha dificultado el trabajo del traductor.

Xuanzi CAO y Montserrat FRANQUESA Estudio de la traducción del chino al español de Sutra del estrado 
También hemos constatado, con respecto a la fraseología, que es imposible que ambos sistemas lingüísticos, chino y español, encajen completamente. El modo de expresión y el pensamiento de ambas culturas son extraordinariamente lejanos. Se pone especial énfasis en el significado original y se descuida, inevitablemente, el peso cultural de las expresiones. Al traductor le resulta más fácil aproximarse a la forma del texto original siendo literal. En el fondo, esto no solo refleja diferencias en la sintaxis, sino también en la gramática y en la estructura general en ambos idiomas.

Respecto al estilo, el chino prefiere escribir con numerosos adornos haciendo que el texto sea elegante pero el significado hermético. En comparación, el español tiende a evitar la repetición. En realidad, esa disimilitud está en la base de la eterna polémica entre 文(Wen) y 质(Zhi). Según Ren Jiyu, en la historia de la traducción de China existen las dos escuelas, la que aboga por la sencillez (质朴) y la que prima la elegancia (文丽) (Ma 2004, 28). Es una controversia que se remonta a tiempos lejanos y sobre la cual se ha debatido y se sigue debatiendo.

El buen traductor debe conocer profundamente la cultura y forma de pensamiento de la lengua original y de la de llegada. En el caso de Sutra del estrado, el original chino se remonta al sánscrito, con lo que constatamos una dificultad añadida. Hasta hoy en día, a pesar del rápido aumento del intercambio cultural entre China y España, escasean los materiales para aprender ambos idiomas y los programas de formación de traductores, a excepción de algunos centrados en ámbitos relacionados con los negocios o la tecnología y que no prestan excesiva atención a la literatura y a la formación de traductores literarios. La obra de Laureano Ramírez no solo incluye el original, sino que también añade un resumen de términos búdicos, que en la práctica constituye un interesante material didáctico para la formación de futuros traductores. En este sentido, el trabajo del traductor ha enriquecido el repertorio de la literatura española en el ámbito del budismo chino. Si lo comparamos con el Sutra del estrado en inglés (Yampolsky 2012), donde se hace referencia a términos frecuentes de la religión budista (tales como 摩诃般若波罗蜜法 [Dharma of the Great Perfection of Wisdom] o 无相戒 [Precepts of formlessness], entre otros) sin que se añada exégesis ni anotación alguna, sino únicamente traducidos de manera literal, en la versión española tanto los términos comunes como los específicos de este ámbito se trasladan con una explicación a pie de página. El budismo en los países hispanohablantes carece de tradición y de la familiaridad que tienen otras religiones, por lo que cualquier enfoque representa una concepción novedosa que debe ser previamente esclarecida.

Como dijo Humboldt ya en 1820, cada lenguaje tiene su particularidad, pero eso no significa que la traducción no sea posible ni que no se pueda traducir de manera efectiva (Humboldt 2011). Entre lenguas lejanas no hay posibilidad de intercomprensión, el trasvase cultural siempre es un reto. La cultura de un país es como un libro enciclopédico que contiene toda la idiosincrasia que se expresa en el lenguaje. Al traducir a cualquier lengua meta, se debe procurar conseguir la transmisión del significado completo y a la vez emplear los métodos adecuados para transmitir el estilo y la fraseología. En el caso de la versión del Sutra del estrado de Ramírez Bellerín, nuestro

Xuanzi CAO Y Montserrat FRANQUESA Estudio de la traducción del chino al español de Sutra del estrado
CLINA

vol. 6-1, June 2020, 109-125

elSSN: 2444-1961

Ediciones Universidad de Salamanca - CC BY-NC-ND 
análisis demuestra que en general consigue superar la distancia cultural, con decisiones apropiadas y que se adaptan a la cultura de llegada.

\section{BIBLIOGRAFÍA}

CHEN, Fukang (陈福康). 2000. 中国译学理论史稿 Materiales para la historia de las teorías traductológicas en China]. Shanghai: Editorial Educación en Lenguas Extranjeras.

CHANG, Liang (常亮) y Cuihua ZHAO (赵翠华). 2018. 《美国的六祖坛经研究.[La investigación de Sutra del Estrado en los Estados Unidos]". Journal of Hebei Normal University for Nationalities, 4 (38): 32-39.

Hul, Neng (惠能). 2000. Sutra del Estrado [orig. 坛经. Traducido por Laureano Ramírez Bellerín. Barcelona: Kairós.

Hul, Neng (惠能). 1990. El Altar Sutra del Sexto Patriarca Zen [orig. 六祖坛经. Traducido por Qianhui Wang (王茜慧). Taipei: Caves Books Ltd.

HUMBOLDT, Wilhelm von. 2011. 论与语言发展的不同时期有关的比较语言问题, [Sobre la lingüística comparativa en relación a las diferentes épocas del desarrollo lingüístico]. Traducción de Yao, Xiaoping. Changsha: Hunan Education Publishing House.

Luo, Xinzhang (罗新璋) y Chen YINGNIAN (陈应年). 2015. 翻译论集 [Antología de textos sobre la traducción]. Beijing: Commercial Press.

MA, Zuyi (马祖毅). 2004. 中国翻译简史 [Breve historia de la traducción en China]. Beijing: China Translation \& Publishing Corporation.

YAmPolsky, Philip. 2012. The Platform Sutra of the Sixth Patriarch: The Text of the Tun-huang Manuscript, with Translation, Introduction, and Notes. Nueva York: Colombia University Press.

YANG, Zengwen (杨曾文). 1988. «世界宗教研究 [Investigación de la religión en el mundo]». 中日 佛教学术会议文集 [Antología del congreso acádemico del budismo entre China y Japón], 6: 104.

ZHANG, Liwen (张立文). 1996. 玄境: 道学与中国文 [Xuanjing: Taoísmo y la cultura china]. Beijing: People's Publishing House.

Xuanzi CAO y Montserrat FRANQUESA

Estudio de la traducción del chino al español de Sutra del estrado 